\title{
REDUCTION OF THE ERROR MULTIPLIER BY A LONG-TERM ANALYSIS OF THE CHARACTERISTIC BEHAVIORS OF PROPORTIONAL COUNTERS
}

\section{INGRID U. OLSSON}

\author{
Department of Physics, Uppsala University, Box 530, S-751 21 Uppsala, Sweden
}

\begin{abstract}
A significant reduction in cosmic-ray activity and backgrounds of the gas-filled proportional counters, as measured in a heavy iron shield, was observed when the Uppsala Conventional ${ }^{14} \mathrm{C}$ Laboratory was moved in 1984 . The new site was better shielded from cosmic rays because of additional concrete layers above the laboratory. A study that lasted over one year yielded a figure for the muon reduction. The backgrounds were reduced approximately to the extent expected from the soft-component contribution at the old Laboratory as judged from barometric-pressure dependence. After a few years, new electronics enforced, and enabled, the revision and improvement of the standard values for the activity and age calculations.

A careful analysis of the results for the counters has increased the accuracy of the small corrections needed to yield internal error-multiplication factors mostly between 1 and 1.5 for the background for short periods of up to 12 months, and $<1.1$ for the oxalic acid samples combined for the last few years of measurements in the laboratory. Similar results were obtained for two counters.
\end{abstract}

\section{INTRODUCTION}

The aim of this investigation was to improve the standard values used for the activity calculations for two gas-filled proportional counters, and in particular to study any pressure dependence, to determine whether there was a relationship between the muons and the background and to learn more about the internal error-multiplication factor.

Late in 1984, the Laboratory was moved from a tower of the Department of Physics to the basement of a building surmounted by three floors and an attic. The electronic system was replaced in 1989. The background was, as expected, lower in the new Laboratory, and the new electronics allowed improved studies of the counting statistics. The muon count rate decreased by almost one quarter.

De Vries $(1956,1957)$ had shown that some of the background of the counters in his laboratory was from the soft component of the cosmic rays. Thus, background decreased with an increase of barometric pressure. De Vries, Stuiver and Olsson (1959) gave a figure, $-0.0035 \pm 0.0010 \mathrm{cpm}$ per $\mathrm{mm}$ $\mathrm{Hg}$ change in barometric pressure, for a background of $c a .0 .9 \mathrm{cpm}$ for the counter in the Uppsala Laboratory (or $\mathrm{ca}$. $-0.003 \mathrm{cpm} \mathrm{mbar}^{-1}$ ). This background dependence on the barometric pressure is too high to be due only to a decrease of the hard component of the cosmic rays $\left(-0.12 \% \mathrm{mbar}^{-1}\right)$. The dependence on the soft (nucleonic) component producing neutrons in the shield is $-0.7 \% \mathrm{mbar}^{-1}$. In Uppsala, the background seemed to be lower for 1 and 2 atm $\mathrm{CO}_{2}$ filling pressure than for $3 \mathrm{~atm}$ (de Vries, Stuiver and Olsson 1959). Olsson (1988) published results indicating that the barometricpressure dependence of the background had probably disappeared or at least declined appreciably, when the total passive shield was improved because of the additional concrete layers above the iron shield in the new laboratory. In consequence, the relation between the background and the muon count rate was also studied. Olsson (1982) summarized oxalic-acid values (activity of the modern standard) for many years, showing that there was a residual pressure dependence of the activity after normalization to standard filling pressure, normalized for temperature variations, and under the same conditions in the electronic system. The decline in the count rate with increasing pressure was significant according to that study.

Olsson et al. (1962) and El-Daoushy and Olsson (1977) similarly studied the characteristics for varying pressures and discriminator levels. At the half-life measurement, extrapolation to the discriminator level 0 was included and the end effects were eliminated with the help of a difference 
counter. This was accomplished by moving one end by turning a carefully calibrated screw. The extrapolation was $c a .1 \%$ and the uncertainty estimated to $0.2 \%$ - one of the largest uncertainties (Olsson et al. 1962, Table 1). El-Daoushy and Olsson (1977) could detect a decreasing background for increasing discriminator levels, and an increasing background for increasing pressure for a counter of the de Vries, Stuiver and Olsson (1959) design but with a nonlinear dependence. Previously de Vries and Barendsen (1953) had shown that there is an optimal discriminator level for each counter determined by the gas pressure, the geometry of the counter and the radiation studied.

\section{METHODS}

The gas-filled counters were placed in a 9-ton shield that incorporated paraffin with boric acid to reduce the influence of neutrons (Olsson 1958, 1988). One of the two counters used in this study, PR4, was placed inside a mercury tank and the other, PR5, outside this tank in the same cavity of the shield. The gamma rays produced at the capture of the neutrons were thus better absorbed for PR4 than for PR5. A set of Geiger counters surrounded the parcel comprising the mercury tank and two copper tubes, attached to the tank, where proportional counters could be placed. The Geigers were arranged (Olsson 1988, Fig. 4) so that muons could not pass the proportional counters without triggering the Geigers. These were connected to a quenching circuit (Caini and Olsson 1962). The new electronics for the proportional counters, including the anticoincidence traps, were designed and described by Björnfot (1990). Three lines, or units, were available, so a line used for recording the decays for the activity measurements for one counter could be used in parallel with another line for comparisons. The discriminator levels were slightly different. Each line was split into three channels with different discriminator levels - one for betas and muons with the same discriminator level, one for alphas and another muon channel with a discriminator level to yield a count rate equivalent to that on the steep part of the characteristic (Olsson 1958) to trace any drift during a measurement. The high-voltage supplies for the proportional counters came from Fluke ${ }^{\circledR}$ (Model 410B). The measurement period could be varied, but usually 100-min periods were chosen so the results for $c a .10$ short periods were recorded every "night". A simple statistical program allowed an automatic analysis of the uncertainties for these periods, giving the ratio between the real spread and that expected on mathematical grounds ( $\sigma_{\text {real }} / \sigma_{\text {expected, }}$ here called the K-value; the term "error multiplier" is also used). This program was based on the principles given in Stuiver (1982).

The purity of the gas was checked on the first day shortly after filling the counter, and the next day before it was refilled, or before the next "overnight" period (the same gas without refilling) was started. For the purity check, muons were found suitable and used $($ Olsson 1958,1988$)$ so that the standard procedure was to take three points on the steep part of the muon characteristic, each $c a .20$ min. The computer calculated the normalized voltage for a fixed, chosen, count rate, "midpoint", after normalization for barometric pressure and the slope, where this part of the characteristic could be assumed to be a straight line. The voltage for the working point was chosen close to a fixed value above the voltage for the midpoint, yielding the effective voltage. The result obtained at the effective voltage was then normalized to the normal working point using the slope on the plateau. To determine this slope, the effective voltage was varied-greatly for the background samples, and less for the oxalic-acid samples, but more than for normal samples. The steep region had a slope of $c a$. 0.20 muons $\min ^{-1} \mathrm{~V}^{-1}$. This number decreased with increasing pressure. The corresponding net count rates on the plateau were $c a .86$ and 81 , respectively. The count rate was thus $c a .42 \pm 1.5 \mathrm{cpm}$ for the midpoint if 20-min measurements were used. The natural variations, the uncertainty, of the muons should be taken into consideration. It could be seen that each point could be measured with an uncertainty corresponding to $c a .10$ volts. 
The program for activity and age calculations was a revised version of that described by Olsson (1966). It allowed normalizations for background variations with barometric pressure and the extra pressure correction for the betas seen by Olsson (1982). Factors for the various corrections could be varied arbitrarily by changing the standard values for each counter. The corresponding filling-pressure correction for the background, determined in this study, was not included in the revised program, however. These corrections had thus to be made "by hand". The uncertainties of the physical measurements of the pressure, ${ }^{13} \mathrm{C}$, determinations of the "mid point" of the steep part and the factors for the various normalizations were considered in the calculations (Olsson 1958; Olsson et al. 1962).

From autumn 1984, the filling pressure was measured using pressure transducers, type PDCR 135/A, connected to a multichannel indicator constructed in the electronic workshop of the Department. It was adjusted to indicate high numbers and not calibrated to yield absolute values although we chose a setting such that $c a .1$ bar corresponded to 500 "pressure units". The pressure values given here are thus in "units", each corresponding to $c a$. 2 mbar. Since the activities of ${ }^{14} \mathrm{C}$ samples should be related to the international standards, there is no need for absolute pressure values in studies such as this. The pressures had, however, to be normalized to a fixed temperature to yield results for the same number of molecules as for the standard. Their measurement in the counter, with a slight correction for the dead volume in the connections, excluded possible error because of adsorption, which can disturb the result if the pressure is measured in a volume outside the shield and adsorbing material is used (Olsson et al. 1962; Olsson 1988: 200-201). The temperatures were measured using flat resistor thermometers fixed on the outer surface of the counters. The pressure was measured $c a .1 \mathrm{~h}$ after the counter was filled to allow temperature stabilization and again just before the gas was removed from the counter or sometimes once a day-when the gas was left in the counter day and night.

The barometric pressure was measured in $\mathrm{mm} \mathrm{Hg}$ with a barograph. (This has been the practice since the Laboratory was established; it was found convenient to continue this procedure for routine measurements and for comparisons between new values and old ones from the previous years.) For very low and very high pressures, results from the Department of Meteorology or from a mercury barometer were used, translated to the Laboratory barograph, since this failed at extreme values.

\section{THE DEPENDENCIES STUDIED}

The dependencies of the background on barometric pressure, effective voltage and filling pressure were studied, as a first approximation, assuming a linear relationship although a nonlinear dependence on filling pressure could be expected (Olsson 1958; El-Daoushy and Olsson 1977). A least squares program written at Uppsala in the 1960 s was used and could be combined with the statistical analysis. Any drift with time and discriminator level was also checked. Since the early measurements (Olsson 1958) also included comparisons of the muon count-rate variations with the cosmicray results from a study group of cosmic rays, and the muons were also used for the purity checks, the muon results were normalized to a standard barometric pressure. The background dependence on these normalized values was also investigated. The dependencies of the measured oxalic-acid activity on the effective voltage, filling pressure, time and discriminator level were also examined assuming a linear relationship.

To start, results from periods of a few months or other suitable periods were used for the long-term statistical analyses. The results for samples from a combustion were also compared with those from other combustions. The short periods were later combined to longer intervals. Certain events, such 
as partial adjustments of the electronics, voltage breaks and problems with the equipment naturally limited the periods considered.

The uncertainty in a dependence calculated using least squares will be considerable if the range is short over which the independent variable is measured. This was often the case for the muons. The weighted means for short periods were used. For the weighting, the calculated value from the least squares calculation was used as well as the $\sigma$-values for the beta channels. These two values were also compared with a median value obtained after exclusion of values with large uncertainties. By a combination of a critical, albeit at first subjective, evaluation of these values, successively prolonged periods and iterations of the activity-calculation program using step-wise improved standard values, the dependencies could finally be excluded or accurately and objectively determined and included in the calculations for the final age and activity measurements from 1989 until 1994.

\section{SAMPLES USED}

Anthracite was pretreated and burned on 10 occasions and split into 13 background samples used in this investigation. Oxalic acid was burned to yield seven samples with internal numbers $11,12,14$, $15,16,17$ and 18 . The oxalic acid used was previously called "Old oxalic acid" (also known as NBS SRM-4990 (Mann 1983), Oxalic Acid I, SRM 4990B, or HOxI (Long 1995)). The $\delta^{13} \mathrm{C}$ values from these seven samples ranged from -21.14 to $-18.35 \%$. Each sample was measured at least twice. From the agreement between the results for a single oxalic-acid sample it is realistic to estimate the uncertainty in each value to $0.2 \%$. The overall uncertainty on oxalic acid is thus here $c a$. $1 \%$, deriving mainly from the combustion. The earlier values from the Uppsala Conventional Laboratory, oxalic-acid numbers 1 to 10 , showed less spread. For these earlier results the overall uncertainty is close to $0.3 \%$. Another source of uncertainty, presumably unlikely for the actual oxalic-acid samples, may derive from fractionation if the aliquot needed is taken from gas just from the sublimation of dry ice. Craig (1961) and Polach (1973) give examples of wide ranges for $\delta^{13} \mathrm{C}$ obtained at different laboratories. Grey et al. (1969), Kim (1970), Polach and Krueger (1973) and Valastro, Land and Varela (1977) report fractionation at the combustion-if not complete.

\section{LIMITATIONS OF THE STATISTICAL ANALYSIS}

The simplified statistical analysis acted as a tool for evaluating any improvements when adjusting the normalization factors. Since the program was devised for variables with the same statistical uncertainty, it was well suited for 100-min results "overnight" although the number of points was only $c a$. 10 or 12 or sometimes, e.g. over weekends, somewhat higher. Only complete 100-min periods were taken into consideration for the $\mathrm{K}$-value calculation. The results from a much shorter period could very well fit the mean value from a statistical point of view, but would sometimes deviate and have such a large 1 $\sigma$-value that, although acceptable, it would cause the K-value to appear much too high. When a few "overnight" periods were combined the short periods were thus also excluded for the K-value calculation although kept for the least squares calculations, for which this limitation was not valid. Results from special tests of a few hours duration could thus be included. It was also seen in reality that most of the $\mathrm{K}$-values tended to decrease when the shortest periods were removed.

The number of the points in the least squares program was also limited to 99 . This was not a serious limitation since it seemed appropriate to divide the interval from 1989 to 1994 into shorter periods.

Because of the shape of the characteristics it was appropriate, and safe, to limit the voltage range to ca. 200 volts for the least squares analysis-sufficient for normal activity measurements. It was 
seen, albeit from too few points to draw definite conclusions, that a wider range could have been used. For the barometric-pressure dependence, the natural variations were $<$ or $\ll 30 \mathrm{~mm} \mathrm{Hg}$ for periods of months. Thus, a 10-mm change with a pressure dependence of $-0.001 \mathrm{cpm}$ per $\mathrm{mm} \mathrm{Hg}$ will affect the background by $0.01 \mathrm{cpm}$ or one third of the statistical uncertainty "overnight", making it difficult to determine this dependence with accuracy unless many values are available. The filling pressure could, however, be varied at whim. Finally, it was observed that the pressure range had to be limited for the oxalic-acid samples because of the complex relationship between such elements as gas amplification, pressure and shape of the characteristics.

\section{RESULTS AND DISCUSSIONS}

\section{Pressure Dependencies}

The background for PR4 did not show any significant relationship with the barometric pressure. The weighted mean value is $-0.000187 \pm 0.000284 \mathrm{cpm}$ per $\mathrm{mm} \mathrm{Hg}$. The corresponding value for PR5 is $-0.0010 \pm 0.0003$, indicating a residual barometric-pressure dependence.

The filling-pressure dependencies for the backgrounds were evaluated to $-0.00007 \mathrm{cpm}$ per pressure unit for PR4 and -0.00009 for PR5.

The decrease of the oxalic-acid activity with increasing pressure, after normalization for the number of molecules in the counter, was significant. The uncertainty of the final figures for this was slightly $<10 \%$ of the values to be used for age calculations. For PR4, the total pressure range had to be split into two parts-one with 126 results at $c a .1360$ units (a pressure close to that used for normal samples), and a second with 52 samples at ca. 1050 units. (One unit is about equivalent to 2 mbar, as stated earlier.) There was a gap of slightly $>100$ units ( $200 \mathrm{mbar}$ ), except for six samples treated together with the low-pressure group, between these two groups. Further, the whole period was divided into two because of a change of electronic units. Oxalic acid 15 was treated separately. Originally, this oxalic acid was thought to be contaminated. The main reason was that the value of the filling pressure deviated significantly from those of the other oxalic acids-especially in one of the counters. The corrections because of the filling-pressure dependence were finally determined for PR4 to -0.00165 unit $^{-1}$ for normal pressures and -0.00092 , with large uncertainty, for low pressures. The K-value for oxalic 15 in the first long period was 0.86 and for the other oxalic acids for the two periods it was 1.11 and 1.25, when the iterations were stopped. A limited voltage range was used. For the low-pressure samples, the corresponding values, although obtained with fewer samples, were $1.24,0.95$ and 1.22 . For PR5, the dependence was determined to be -0.0010 per pressure unit, and only one range, $c a .700$ units, was used because of the good $\mathrm{K}$-value, e.g., 1.04, for 90 results. Ten samples, oxalic-acid 15, with a range of $c a$. 580 units, were treated separately, yielding a Kvalue of 0.98 for this counter. The pressure-dependence thus affects the result slightly more than the statistical uncertainty for "overnight" periods for a deviation of $c a .60$ or 100 units, respectively.

\section{Statistical Spread}

The available "overnight" background K-values are 157 for PR4 and 174 for PR5 with 76 and 89 values $<1.0$, respectively. The values for oxalic acid are 126 and 78 with 71 and 32 values $<1.0$.

The background measurements for PR4 yielded K-values 1.81, 1.32 and 1.34 for three periods. The values for PR5, when split into four short periods with a total of 74 "overnight" values and two longer intervals with 36 and 90 values, are 1.48, 1.18, 1.55 and 1.84 for the shorter, and 1.17 and 1.19 for the longer series. 
It was impossible to determine whether any relationship existed between the backgrounds and muons, either in the Geigers or in the proportional counters.

\section{Comments on the Values}

The K-values for each of the seven oxalic-acid samples during the first and second periods with normal pressure in PR4 and for the entire single period in PR5 varied from 0.77 to 1.41. A slight increase of the background of PR4, from $0.9435 \pm 0.0031$ to $0.9518 \pm 0.0040 \mathrm{cpm}$, observed from the day the electronic unit (no. 3) had to be replaced (by no. 2) is probably due to slight differences in the two units. The oxalic-acid activity increased on this occasion by $c a .0 .6 \pm 0.3 \%$. The calculated uncertainty before and after the replacement was 0.15 and $0.18 \%$, respectively.

No relationship between the activities of the different oxalic acids and the $\delta^{13} \mathrm{C}$ values, or the varying yield at the combustions, could be detected. No systematic activity differences between the different oxalic-acid samples were observed.

\section{CONCLUSION}

Simple, old-fashioned, cheap equipment can yield reliable results and prove to be stable for many years. A simple statistical analysis can be applied for quality control as well as results from the international studies, e.g., on material from IAEA. This study indicates that the error multiplier can be kept as low as 1.1.

\section{ACKNOWLEDGMENTS}

The author is very grateful for the continuous support received from the Swedish Natural Science Research Council during her employment in the Laboratory and from the laboratory engineers who helped her with the measurements covered by this investigation from 1989 until 1994.

\section{REFERENCES}

Björnfot, L. 1990 A computer based data acquisition system for C-14 measurements. Fil. lic. thesis. Uppsala University Department of Physics 1221: 1-60.

Caini, V. and Olsson, I. U. 1962 A quenching circuit for self-quenching Geiger-Müller arrays. Arkiv för Fysik 22: 225-235.

Craig, H. 1961 Mass-spectrometer analyses of radiocarbon standards. Radiocarbon 3: 1-3.

de Vries, Hl. 1956 Cosmic radiation during the solar flare of February $23^{\mathrm{d}}$ and its effect on ${ }^{14} \mathrm{C}$ age measurements. Physica 22: 357.

1957 Further analysis of the neutron component of the background of counters used for ${ }^{14} \mathrm{C}$ age measurements. Nuclear Physics 3: 65-68.

de Vries, $\mathrm{Hl}$ and Barendsen, G. W. 1953 Radio-carbon dating by a proportional counter filled with carbondioxide. Physica 19: 987-1003.

de Vries, Hl., Stuiver, M. and Olsson, I. U. 1959 A proportional counter for low level counting with high efficiency. Nuclear Instruments and Methods in Physics Research 5: 111-114.

El-Daoushy, M. F. A. F. and Olsson, I. U. 1977 An improved proportional counter for low-level counting with high efficiency. In Povinec, P. and Usacev, S., eds., Low Radioactivity Measurements and Applications: Proceedings of the International Conference, The High Tatras, CSSR, 6-10 October 1975. Bratislava, Slovenske Pedagogicke Nakladatelstvo: 85-90.

Grey, D. C., Damon, P. E., Haynes, C. V. and Long, A. 1969 Carbon-isotope fractionation during wet oxidation of oxalic acid. Radiocarbon 11: 1-2.

Kim, S. M. 1970 Wet oxidation of oxalic acid used in radiocarbon dating and ${ }^{14} \mathrm{C}$ fractionation during the oxidation. Journal of the Korean Chemical Society 14: 49-50.

Long, A. 1995 From the Editor. Radiocarbon 37(1): iii-iv.

Mann, W. B. 1983 An international reference material for radiocarbon dating. In Stuiver, M. and Kra, R. S., eds., Proceedings of the 11 th International ${ }^{14} \mathrm{C}$ Conference. Radiocarbon 25(2): 519-527.

Olsson, I. $1958 \mathrm{~A} \mathrm{C}^{14}$ dating station using the $\mathrm{CO}_{2}$ proportional counting method. Arkiv för Fysik 13: 37-60.

Olsson, I. U. 1966 Computer calculations of $\mathrm{C}^{14}$ determinations. In Radiocarbon and Tritium Dating. Proceedings of the 6th International Conference on Radiocarbon and Tritium Dating, Washington State University, 
Pullman, Washington, June 7-11, 1965. Oak Ridge, Tennessee, U.S. Atomic Energy Commission: 383392.

1982 The long-term stability of a proportional counter filled with $\mathrm{CO}_{2}$. In Povinec, P., ed., Low Level Counting: Proceedings of the Second International Conference, Low Radioactivities '80, The High Tatras. Physics and Applications 8. Bratislava, VEDA: $145-153$.

1988 Low-level counting using gas-filled counters as applied to ${ }^{14} \mathrm{C}$ dating with emphasis on reliability. In Garcia-Leon, M. and Madurga, G., eds., Low-Level Measurements and Their Applications to Environmental Radioactivity. Proceedings of the First International Summer School, La Rábida, Huelva, Spain. Singapore, World Scientific Publishing Company: 171223.

Olsson, I. U., Karlén, I., Turnbull, A. H. and Prosser, N.
J. D. 1962 A determination of the half-life of $\mathrm{C}^{14}$ with a proportional counter. Arkiv för Fysik 22: 237-255.

Polach, H. 1973 Cross checking of NBS oxalic acid and secondary laboratory radiocarbon dating standards. In Rafter, A. T. and Grant-Taylor, T., eds., Proceedings of the 8th International ${ }^{14} \mathrm{C}$ Dating Conference. Wellington, Royal Society of New Zealand: 688-717.

Polach, H. A. and Krueger, H. A. 1973 Isotope fractionation of NBS oxalic acid and ANU-sucrose radiocarbon dating standards. In Rafter, A. T. and Grant-Taylor, T., eds., Proceedings of the 8th International ${ }^{14} \mathrm{C}$ Dating Conference. Wellington, Royal Society of New Zealand: 718-727.

Stuiver, M. 1982 A high-precision calibration of the AD radiocarbon time scale. Radiocarbon 24(1): 1-26.

Valastro, S., Jr., Land, L. S. and Varela, A. G. 1977 An improved procedure for wet oxidation of the 14C NBS oxalic acid standard. Radiocarbon 19(3): 375-382. 